\title{
DeepPVP: phenotype-based prioritization of causative variants using deep learning
}

\author{
Imane Boudellioua ${ }^{1,2}$, Maxat Kulmanov1,2, Paul N. Schofield ${ }^{3}$, Georgios V. Gkoutos 4,5,6,7,8,9
}

and Robert Hoehndorf ${ }^{1,2^{*}}$ (D)

\begin{abstract}
Background: Prioritization of variants in personal genomic data is a major challenge. Recently, computational methods that rely on comparing phenotype similarity have shown to be useful to identify causative variants. In these methods, pathogenicity prediction is combined with a semantic similarity measure to prioritize not only variants that are likely to be dysfunctional but those that are likely involved in the pathogenesis of a patient's phenotype.

Results: We have developed DeepPVP, a variant prioritization method that combined automated inference with deep neural networks to identify the likely causative variants in whole exome or whole genome sequence data. We demonstrate that DeepPVP performs significantly better than existing methods, including phenotype-based methods that use similar features. DeepPVP is freely available at https://github.com/bio-ontology-research-group/ phenomenet-vp.

Conclusions: DeepPVP further improves on existing variant prioritization methods both in terms of speed as well as accuracy.
\end{abstract}

\section{Keywords: Variant prioritization, Phenotype, Machine learning, Ontology}

\section{Background}

There is now a large number of methods available for prioritizing variants in whole exome or whole genome datasets [1]. These methods commonly identify the variants which are pathogenic, i.e., the variants that may alter normal functions of a protein, either directly through a change in a protein's amino acid sequence or indirectly through a change of expression [2-4]. In coding and noncoding DNA sequences, there are usually multiple variants that could possibly be pathogenic, but most of them are sub-clinical or will not result in any detectable phenotypic manifestations [5].

Recently, several methods have become available that utilize information about phenotypes observed in a patient to identify potentially causative variants [6-9].

*Correspondence: robert.hoehndorf@kaust.edu.sa

${ }^{1}$ Computational Bioscience Research Center (CBRC), King Abdullah University of Science and Technology, 4700 KAUST, 23955-6900 Thuwal, Kingdom of Saudi Arabia

${ }^{2}$ Computer, Electrical and Mathematical Sciences \& Engineering Division (CEMSE), King Abdullah University of Science and Technology, 4700 KAUST, PO Box 2882, 23955-6900 Thuwal, Kingdom of Saudi Arabia

Full list of author information is available at the end of the article
Phenotypes are useful for identifying gene-disease associations because they implicitly reflect interactions occurring within an organism across multiple levels of organisation [10-12]. Phenotype-based methods work by comparing the phenotypes of a patient with a knowledgebase of gene-to-phenotype associations. A measure of phenotypic similarity is computed between a patient's phenotypes and abnormal phenotypes associated with gene variants or mutations. The phenotypic similarity is then used either as a filter to remove pathogenic variants in genes that are not associated with similar phenotypes to the ones observed in the patient [9] or as a feature in machine learning approaches $[6,7]$.

The gene-to-phenotype associations used in phenotypebased prioritization strategies come from clinical observations such as those reported in the Online Mendelian Inheritance in Man (OMIM) database [13] or in the ClinVar database [14]. In some cases, they may also come from model organisms. Comparing model organism phenotypes to human phenotypes (i.e., the phenotypes observed in a patient) requires a framework that allows phenotypes of different species to be compared, such as the Uberpheno [15] or PhenomeNET ontology [16].

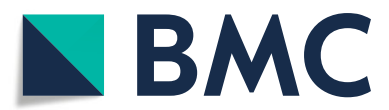

(c) The Author(s). 2019 Open Access This article is distributed under the terms of the Creative Commons Attribution 4.0 International License (http://creativecommons.org/licenses/by/4.0/), which permits unrestricted use, distribution, and reproduction in any medium, provided you give appropriate credit to the original author(s) and the source, provide a link to the Creative Commons license, and indicate if changes were made. The Creative Commons Public Domain Dedication waiver (http://creativecommons.org/publicdomain/zero/1.0/) applies to the data made available in this article, unless otherwise stated. 
We have previously developed the PhenomeNET Variant Predictor (PVP) [7] to prioritize causative variants in personal genomic data. We have shown that PVP outperforms other phenotype-based approaches such as the Exomiser or Genomiser tools [17, 18], or Phevor [9]. PVP is based on a random forest classifier, similarly to Exomiser and Genomiser, which also use a random forest. Features used to classify a variant as causative or non-causative combine a phenotype similarity score (to prioritize a gene as being associated with the phenotypes observed in the patient) and a pathogenicity score, as well as other features such as the mode of inheritance and genotype of the variant. As most variants are neutral, there is a very large imbalance between positive and negatives, and the challenges for building a machine learning model for finding causative variants is to account for this imbalance during training and testing.

Recently, deep neural networks have shown to be successful in many domains [19] and often result in better classification performance [4]. We have developed DeepPVP, an extension of the PVP system which uses deep learning and achieves significantly better performance in predicting disease-associated variants than the previous PVP system, as well as competing algorithms that combine pathogenicity and phenotype similarity. DeepPVP not only uses a deep artificial neural network to classify variants into causative and non-causative but also corrects for a common bias in variant prioritization methods $[20,21]$ in which gene-based features are repeated and potentially lead to overfitting. DeepPVP is freely available at https://github.com/bio-ontology-research-group/ phenomenet-vp.

\section{Implementation}

Training and testing data

We downloaded the ClinVar database 7th Feb, 2017, and extracted GRCh37 genomic variants annotated with at least one disease from OMIM, characterized as Pathogenic in their clinical significance, and not annotated with conflicting interpretation in their review status. We obtained 31,156 pathogenic variants associated with 3938 diseases in total and the set of these variants constitutes candidate positive instances in our training dataset. We also extracted GRCh37 genomic variants that are characterized as Benign in clinical significance, and not annotated with conflicting interpretation in their review status. We obtained 23,808 such benign genomic variants from ClinVar which form the candidate negative instances in our training dataset. We excluded any variant records mapped to more than one gene and variant records with missing information on the reference or alternate alleles. For pathogenic variant records, we defined variantdisease pairs for each pathogenic variant and its associated OMIM disease. In our dataset, some pathogenic variants are annotated with multiple OMIM diseases. For each of these variants and the $n$ OMIM diseases they may cause, we created $n$ variant-disease pairs. For example, variant rs201108965 in TMEM216 is annotated with two diseases; Joubert syndrome 2 (OMIM:608091) and Meckel syndrome type 2 (OMIM : 603194). We define two variant-disease pairs (rs201108965, OMIM : 608091) and (rs201108965, OMIM: 603194). After this step, we have 30,770 pathogenic variant-disease pairs and 20,174 benign variants.

In DeepPVP, we use the zygosity of a variant as one of the training features. The zygosity information is not provided in ClinVar. In a given Variant Call Format (VCF) [22] file, zygosity is represented in the genotype field. For instance, a heterozygous variant will have a genotype value $0 / 1$, while a homozygous variant will have a genotype value $1 / 1$ in the VCF file. We assigned the genotype information to our pathogenic variant-disease pairs based on the mode of inheritance associated with the disease caused by the variant. We extracted the mode of inheritance of the associated OMIM disease using the information provided by the Human Phenotype Ontology (HPO) annotations of OMIM diseases [23]. If the disease's mode of inheritance is recessive, we assigned the zygosity of the variant as homozygote (denoted with genotype 1/1). In this case, we created a variant-disease-zygosity triple representing this information. If the OMIM disease's mode of inheritance is not recessive (i.e., any other mode of inheritance, including dominant, unknown, X-linked, etc.), we generated two variant-disease-zygosity triples and characterized one of them as homozygote (denoted with genotype 1/1) and another as heterozygote (denoted with genotype $0 / 1$ ). For example, pathogenic variant rs397704705 in AP5Z1 is associated with Spastic paraplegia 48 (OMIM : 613647). This OMIM disease is recessive and, hence, we characterize variant $r s 397704705$ with genotype $1 / 1$, generating a variant-disease-zygosity triple consisting of variant rs397704705, disease OMIM : 613647, and genotype $1 / 1$. Another example is the pathogenic variant rs387907031 in ARHGAP31 associated with Adams-Oliver syndrome 1 (OMIM : 100300 ). This disease is dominant and, hence, we generated two variant-disease-zygosity triples: variant rs387907031, disease OMIM:100300, and the genotype $0 / 1$, and variant rs387907031, disease OMIM: 100300 , and genotype $1 / 1$. Since benign variants are not associated with a disease or mode of inheritance, we treat each of them as both a homozygote and heterozygote, generating two variant-zygosity pairs for each benign variant. After this step, we obtained 61,540 triples consisting of pathogenic variant, disease, and zygosity, and 40,348 pairs of benign variant and zygosity.

The triples consisting of variant, disease, and zygosity constitute positive samples. For each positive instance 
$(V, D, Z)$ consisting of a variant, disease, and zygosity, we randomly select, with equal probability, one of two possible negative instances: a randomly selected benign variant in the same gene as $V$, or a triple $\left(V, D^{\prime}, Z\right)$ where $D^{\prime} \neq D$. To map intergenic variants to genes, we link variants to their nearest gene.

For example, a positive instance in our training data is a pathogenic variant rs267606829 in FOXRED1, associated with Mitochondrial complex I deficiency (OMIM : 252010), as a homozygote. A negative instance according to the first strategy could be a benign variant, such as rs1786702, in FOXRED1, as a heterozygote. A negative instance according to the second strategy is the same pathogenic variant, $r$ s267606829 in FOXRED1, as a homozygote, but associated with another OMIM disease such as Tooth Agenesis (OMIM:604625). The resulting training data is balanced.

As an independent and unseen evaluation dataset, we downloaded all variants from ClinVar released between Feb 8th 2017 and Jan 27th 2018. In this dataset, we processed all GRCh37 variants in the same manner as for our training dataset to construct triples of pathogenic variants, disease, and zygosity. However, if the OMIM disease's mode of inheritance of the variant is not recessive (i.e., any other mode of inheritance, including dominant, unknown, X-linked, etc.), we assigned the zygosity randomly either as homozygote (denoted with genotype $1 / 1$ ), or heterozygote (denoted with genotype $0 / 1$ ). We obtained a total of 5686 such triples associated with 1370 diseases for validation.

\section{Generation of synthetic patients}

In our evaluation, we generated a set of synthetic patients as a realistic evaluation case, similarly to previous work $[7,18]$. We randomly selected a whole exome from the 1000 Genomes project [24] and inserted a pathogenic variant $V$, assign the disease associated with $V$ in ClinVar to the exome, and present $V$ as a homo- or heterozygote based on the mode of inheritance associated with the disease. Each of these exomes together with the disease's phenotypes and mode of inheritance form a synthetic patient in which we aim to recover the inserted variant.

\section{Annotating variants}

Annotating variants with pathogenicy scores from CADD [3], DANN [25], and GWAVA [26] is a time-consuming process in PVP [7] and other phenotype-based variant prioritization tools [18], especially when analyzing WGS data comprised of millions of variants. PVP 1.0 uses tabix [27] for indexing and retrieval of the pathogenicty scores per chromosome and genomic position. To optimize the annotation phase of DeepPVP, we extracted 31,491,995 variants from samples of the 1000 Genomes Project [24] and annotated them with pathogenicity scores from
CADD, DANN, and GWAVA. DeepPVP keeps this set of pre-annotated variants in memory to provide fast retrieval of annotations for common variants. DeepPVP utilizes tabix only when the variant annotated is not available in the pre-annotated library, and therefore minimizes disk access.

\section{Model and availability}

We implemented our DeepPVP deep neural network in Python 2.7. We used Keras [28] with a TensorFlow backend [29]. We used one hot encoding to represent our categorical feature of the inheritance mode of the disease. We handled missing values for CADD, GWAVA, DANN, and semantic similarity scores by mean imputation. We also added additional flags for missing values as features. We retrieved gene-phenotype association data from human and model organisms (mouse and zebrafish) on Feb 7th, 2017 and used them to generate the ontology and high level phenotypes and semantic similarity score features.

We used the Hyperas [30] Python library for tuning the hyperparameters of the neural network using the treestructured Parzen estimator (TPE) algorithm [31]. We selected the following hyperparameters for tuning: number of hidden layers (two, three, or four), number of hidden units in each layer $(32,64,67,128,134,201,256$, and $512)$, and the batch size $(2500,5000,10,000,15,000$, and $20,000)$. The hyperparameters combination resulting in best performing model out of 50 trials using Hyperas was selected for the final model setup. Therefore, we designed a sequential model with an input layer, three hidden layers of 67, 32, 256 neurons respectively with Rectified Linear Units (ReLU) [32] activation function, and an output layer with a sigmoid activation function. We trained the model using the Adam optimization algorithm [33] which has been widely adopted for deep learning as a computationally efficient, fast convergent, extension to stochastic gradient descent. We used dropout [34] between the hidden layers and the output layer to prevent overfitting. We trained our DeepPVP model for 100 epochs, 2500 batch size, and a learning rate of 0.001 . In training, we specified a $20 \%$ random stratified-by-disease validation set, and binary cross entropy loss function. We kept the rest of the parameters in their default values. The model was trained on the CPU.

The DeepPVP system (version 2.1), training and evaluation experiments, the synthetic genome sequences and our analysis results can be found at https://github.com/ bio-ontology-research-group/phenomenet-vp.

\section{Results}

\section{DeepPVP: phenotype-based prediction using a deep} artificial neural networks

We developed the Deep PhenomeNET Variant Predictor (DeepPVP) as a system to identify causative variants for 
patients based on personal genomic data as well as phenotypes observed in the patient. We consider a variant to be causative for a disease $D$ if the variant is both pathogenic and affects a structure or function that leads to $D$. This distinction is motivated by the observation that healthy individuals can have multiple highly pathogenic variants resulting in a complete loss of function; it is therefore not usually sufficient to identify pathogenic variants alone as there may be many.

DeepPVP is a command-line tool which takes a Variant Call Format (VCF) [22] file as an input together with a set of phenotypes coded either through the Human Phenotype Ontology (HPO) [35] or the Mammalian Phenotype Ontology (MP) [36]. It outputs a prediction score for each variant in the VCF file; the prediction score measures the likelihood that a variant is causative for the phenotypes specified as input to the method.

To predict whether a variant is causative or not, DeepPVP uses similar features as the PVP system [7] and combines multiple pathogenicity prediction scores, a phenotype similarity computed by the PhenomeNET system, and a high-level phenotypic characterization of a patient. The full list of features used by DeepPVP is listed in Additional file 1: Table S1. All features can be generated from a patient's VCF file and a set of phenotypes coded either with HPO or MP.

In DeepPVP, we use a deep neural network to classify variants as causative or non-causative. Specifically, DeepPVP uses a feed forward neural network with five layers (see Fig. 1). The input layer in our architecture consists of 67 neurons (for the 67 features) and an output layer consisting of a single output neuron which outputs the prediction score of DeepPVP. DeepPVP uses three hidden layers with 67, 32, and 256 neurons, respectively. Each hidden layer uses a Rectified Linear Unit (ReLU) [32] activation function, and the output layer uses a sigmoid activation function.

DeepPVP is trained similarly as PVP to improve performance of identifying causative variants in real genomic sequences (in contrast to performance on a testing set). When training DeepPVP, we use as positive instances all causative variants from our training set together with the phenotypes of the disease for which they are causative. We discriminate these from two kinds of negatives: benign variants (i.e., variants that do not alter protein function) and pathogenic but non-causative variants. We consider pathogenic non-causative variants as pathogenic variants (in our training set) which are not associated with phenotypes of the disease they cause, but rather with a different disease. The aim of this selection strategy is to discriminate causative variants from all other variants.

We train the DeepPVP model using back-propagation, using binary cross entropy as loss function, and evaluate the model's results on predicting causative variants, and compare against several competing methods. While the different evaluation scenarios omit some parts of the information about variants and the diseases they are associated with in order to not bias the evaluation results, we finally retrain a model using all available information and make it available as the final DeepPVP prediction model.

\section{Evaluating DeepPVP's ability to find causative variants}

We use a nested cross-validation experiment as our main evaluation and as means to optimize hyperparameters of our DeepPVP model. We first split our training instances into five folds ( $80 \%$ for training and $20 \%$ for testing) where each fold is stratified by disease (i.e., the diseases are disjoint between all five folds). We use the training part in each of these folds for optimizing parameters and hyperparameters and use the $20 \%$ to evaluate and report the

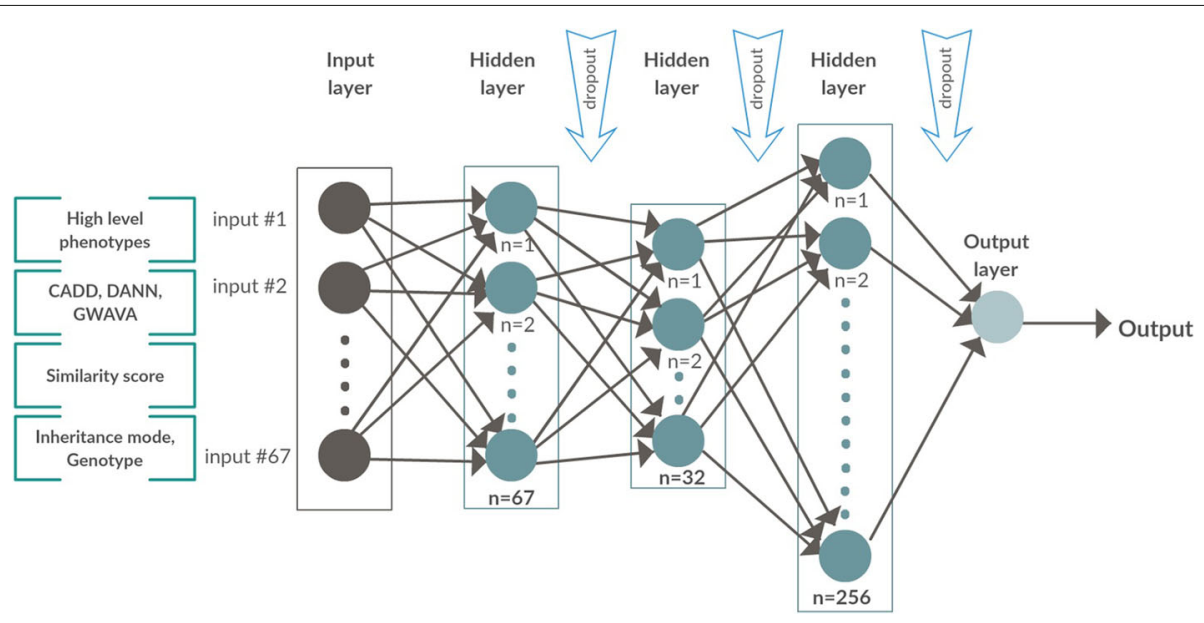

Fig. 1 The DeepPVP neural network model 
final performance. In each of the resulting five folds, we split the $80 \%$ training set into further five folds that are similarly stratified by disease. In total, we end up with 25 different training sets (in the second level of this nested cross-validation) and evaluation sets.

For each of the 25 different splits of data, we tuned the hyperparameters of the network (number of hidden layers, number of hidden units, and batch size) using the Hyperas library [30]. We generated 50 trial models with 50 epochs for each model using the tree-structured Parzen estimator algorithm [31] to find optimal hyperparameters. We ended up with 25 optimized hyperparameters, and we selected the hyperparameters that resulted in the best performance in most of the folds.

After hyperparameter optimization, we train five different models using the optimal set of hyperparameters obtained in the second level of the cross-validation and evaluate the predictive performance of the model on the $20 \%$ set that has not been used for optimizing hyperparameters. The resulting accuracy of our model in 5-fold cross-validation is 0.911 , area under the receiver operating characteristic curve (ROCAUC) [37] is 0.959 , and the area under the precision-recall curve (AUPR) is 0.954 . For comparison, we also performed the same training and evaluation steps using a random forest classifier, and we obtained an accuracy of 0.898 , a ROCAUC of 0.958 , and an AUPR of 0.952.

To accurately evaluate the performance of DeepPVP on real sequencing data, we apply DeepPVP to causative variants added to the ClinVar database on or after Feb 7th, 2017 while our training data is restricted to the variants that have been added to ClinVar before this date. Between Feb 7th, 2017 and Feb 6th, 2018, there were 5686 causative variants added to ClinVar, covering 1370 diseases. 297 of these diseases were not present in our training data. Evaluation on completely unseen variants allows us to estimate under more realistic conditions how well DeepPVP is able to prioritize novel variants.

We generated synthetic patient exomes by inserting a single causative variant from the set of 5686 variants in a randomly selected exome from the 1000 Genomes Project [24] (removing all variants with Minor Allele Frequency (MAF) greater than 1\% (using the frequencies provided by the 1000 Genomes across all populations). We then assign the phenotypes associated with the causative variant in ClinVar, as well as the mode of inheritance of the disease, to the synthetic exome and consider this combination a synthetic patient. We use DeepPVP to prioritize variants given the synthetic patient's filtered VCF file, phenotypes, and mode of inheritance, and determine the rank at which the causative (inserted) variant is found. For comparison, we use PVP v1.1 [7] as well as the random forest classifier we trained using the same training setup as DeepPVP (named DeepPVP-RF). We further compare the performance to the Exomiser version 7.2.1 released on Feb 6th, 2017 with and without using CADD scores as feature. Furthermore, we compare the performance against CADD [3], DANN [25], and GWAVA [26]. Table 1 shows the evaluation results. We find that DeepPVP has an improved performance compared to the original PVP, the use of a neural network classifier gives better results than the random forest classifier, and DeepPVP outperforms Exomiser, CADD, DANN, and GWAVA in this evaluation.

Of the 5686 "new" variants in our ClinVar evaluation set, 5489 are in 934 genes which are associated with phenotypes. These 5489 variants are associated with 1289 diseases. Only 197 variants are in 74 novel genes and are associated with 89 diseases. We test the performance of DeepPVP separately on these 197 variants. DeepPVP identifies 46 of the 197 variants (23\%) at rank one, and 87 variants $(44 \%)$ in the first ten ranks. In comparison, Exomiser and CADD identified 26 and 13 variants at the first, and 61 and 51 variants in the top ten ranks, respectively. Exomiser identified 27 at rank one, and 64 in the top ten ranks. This evaluation demonstrates that DeepPVP can not only identify variants in known disease-associated genes but also in novel genes, although with lower performance than if the gene is already known. While the predictive performance of DeepPVP in this evaluation is lower than in the other types of evaluation, DeepPVP still improves over established methods such as CADD and Exomiser.

Our performance results demonstrate that DeepPVP can identify causative variants with significantly higher recall at rank one and rank ten than several other methods to which we compare, including the original PVP system [7] from which DeepPVP is derived. In some applications of variant prioritization, it is also important to identify causative variants quickly and with low computational costs. We therefore benchmarked the time it takes DeepPVP to process large VCF files. We used a machine equipped with 128 GB Memory and an Intel Xeon ES-2680 v3 CPU with $2.50 \mathrm{GHz}$ and 16 cores, using

Table 1 Comparison of top ranks of ClinVar variants as recovered from WES data; variants with MAF > $1 \%$ are filtered

\begin{tabular}{llllll}
\hline & Top hit & Top 10 hits & Total & ROC AUC & AUPR \\
\hline DeepPVP & $4060(71.40 \%)$ & $4750(83.54 \%)$ & 5686 & 0.94 & 0.66 \\
DeepPVP-RF & $3520(61.91 \%)$ & $4321(75.99 \%)$ & 5686 & 0.95 & 0.55 \\
PVP 1.1 & $3619(63.65 \%)$ & $4076(71.68 \%)$ & 5686 & 0.95 & 0.55 \\
Exomiser & $2910(51.18 \%)$ & $3608(63.45 \%)$ & 5686 & 0.89 & 0.43 \\
Exomiser-CADD & $2926(51.46 \%)$ & $3621(63.68 \%)$ & 5686 & 0.89 & 0.43 \\
CADD & $1060(18.64 \%)$ & $2429(42.72 \%)$ & 5686 & 0.94 & 0.14 \\
DANN & $170(2.99 \%)$ & $1322(23.25 \%)$ & 5686 & 0.90 & 0.03 \\
GWAVA & $63(1.11 \%)$ & $264(4.64 \%)$ & 5686 & 0.66 & 0.01 \\
\hline
\end{tabular}


a 64-bit Ubuntu 16.04 system. We selected a genome from the Personal Genome Project (PGP) [38] which contains $4,120,185$ variants to benchmark DeepPVP. We prioritized variants in this genome using DeepPVP ten times and recorded the time elapsed. On average, analysis of all the variants in the whole genome VCF file took DeepPVP $85 \mathrm{~min}$, i.e., approximately 1.3 milliseconds per variant. Analyzing the same VCF file using the phenotype-based Exomiser software (with and without CADD annotations) took 189 min without CADD annotations (approximately 2.7 milliseconds per variant) and $800 \mathrm{~min}$ with CADD annotations (approximately 11.6 milliseconds per variant).

\section{Conclusions}

DeepPVP is an easy to use and fast phenotype-based tool for prioritizing variants in personal whole exome or whole genome sequence data. DeepPVP takes a VCF file of an individual as input, together with an ontology-based description of the phenotypes observed in an individual. It then aims to identify the variants of the individual that are causative of the phenotypes observed.

Through the use of a deep neural network and an updated training and evaluation strategy, DeepPVP improves over its predecessor PVP, and further outperforms several established methods for variant prioritization, including the phenotype-based tool Exomiser [17, 18] and pathogenicity scoring algorithms such as CADD [3]. Importantly, DeepPVP shows a better performance than other methods in finding variants in novel genes, i.e., genes not previously associated with a disease phenotype, and may therefore be particularly suited for investigating variants in orphan diseases as well as variants of unknown significance in genes not yet associated with phenotypes.

We update DeepPVP in regular intervals when new training data (i.e., variants associated with diseases and phenotypes, as well as gene-phenotype associations) becomes available. DeepPVP is freely available at https://github.com/bio-ontology-research-group/ phenomenet-vp.

\section{Availability and requirements}

- Project name: DeepPVP

- Project home page: https://github.com/bio-ontologyresearch-group/phenomenet-vp

- Operating system: Java virtual machine

- Programming language: Java, Groovy, Python

- Other requirements: none

- License: 4-clause BSD-style license

\section{Additional file}

Additional file 1: Features used to train DeepPVP. A table consisting of the features and their representation used in the training and prediction of DeepPVP. (PDF 33 kb)

\section{Abbreviations}

AUPR: Area under precision-recall curve; CADD: Combined annotation dependent depletion; DeepPVP: Deep PhenomeNet variant predictor; DeepPVP-RF: Deep PhenomeNet variant predictor (random forest); GWAVA: Genome wide annotation of variants; HPO: Human phenotype ontology; MAF: Minor allele frequency; MP: Mammalian phenotype ontology; OMIM: Online mendelian inheritance in man; PGP: Personal genome project; Phevor: Phenotype driven variant ontological re-ranking tool; PVP: PhenomeNet variant predictor; ReLU: Rectified linear units; ROCAUC: Receiver operating characteristic area under the curve; VCF: Variant call format; WES: Whole exome sequencing; WGS: Whole genome sequencing

\section{Funding}

This work was supported by funding from King Abdullah University of Science and Technology (KAUST) Office of Sponsored Research (OSR) under Award No. URF/1/3454-01-01, FCS/1/3657-02-01 and FCC/1/1976-08-01. GVG acknowledges support from H2020-EINFRA (731075) and the National Science Foundation (IOS:1340112) as well as support from the NIHR Birmingham ECMC, NIHR Birmingham SRMRC and the NIHR Birmingham Biomedical Research Centre and the MRC HDR UK. The views expressed in this publication are those of the authors and not necessarily those of the NHS, the National Institute for Health Research, the Medical Research Council or the Department of Health.

\section{Availability of data and materials}

All data and materials, including software developed, is freely available from https://github.com/bio-ontology-research-group/phenomenet-vp.

\section{Authors' contributions}

$\mathrm{IB}$ and RH conceived of the use of a deep neural network, IB and MK implemented DeepPVP, IB trained the models, performed experiments, and evaluated DeepPVP's performance, IB, PNS, GVG, RH interpreted results and critically evaluated the performance, all authors contributed to writing the manuscript. All authors have read and approved the final version of the manuscript.

\section{Ethics approval and consent to participate}

Not applicable.

\section{Consent for publication}

Not applicable.

\section{Competing interests}

The authors declare that they are currently applying for a patent relating to the content of the manuscript.

\section{Publisher's Note}

Springer Nature remains neutral with regard to jurisdictional claims in published maps and institutional affiliations.

\section{Author details}

${ }^{1}$ Computational Bioscience Research Center (CBRC), King Abdullah University of Science and Technology, 4700 KAUST, 23955-6900 Thuwal, Kingdom of Saudi Arabia. ${ }^{2}$ Computer, Electrical and Mathematical Sciences \& Engineering Division (CEMSE), King Abdullah University of Science and Technology, 4700 KAUST, PO Box 2882, 23955-6900 Thuwal, Kingdom of Saudi Arabia.

${ }^{3}$ Department of Physiology, Development \& Neuroscience, University of Cambridge, Downing Street, CB2 3EG Cambridge, UK. ${ }^{4}$ College of Medical and Dental Sciences, Institute of Cancer and Genomic Sciences, Centre for Computational Biology, University of Birmingham, B15 2TT Birmingham, UK. ${ }^{5}$ Institute of Translational Medicine, University Hospitals Birmingham, NHS Foundation Trust, B15 2 TT Birmingham, UK. ${ }^{6} \mathrm{NIHR}$ Experimental Cancer Medicine Centre, B15 2TT Birmingham, UK. ${ }^{7} \mathrm{NIHR}$ Surgical Reconstruction and Microbiology, B15 2TT Birmingham, UK. ${ }^{8}$ NIHR Biomedical Research Centre, B15 $2 \pi$ Birmingham, UK. ${ }^{9} \mathrm{MRC}$ Health Data Research UK, B15 2TT Birmingham, UK.

Received: 29 March 2018 Accepted: 17 January 2019

Published online: 06 February 2019 


\section{References}

1. Eilbeck K, Quinlan A, Yandell M. Settling the score: variant prioritization and mendelian disease. Nat Rev Genet. 2017;18(10):599-612. https://doi. org/10.1038/nrg.2017.52.

2. Huang Y-FF, Gulko B, Siepel A. Fast, scalable prediction of deleterious noncoding variants from functional and population genomic data. Nat Genet. 2017;49(4):618-24

3. Kircher M, Witten D, Jain P, O'Roak B, Cooper G, Shendure J. A general framework for estimating the relative pathogenicity of human genetic variants. Nat Genet. 2014;46(5):310-5. https://doi.org/10.1038/ng.2892.

4. Quang D, Chen Y, Xie X. Dann: a deep learning approach for annotating the pathogenicity of genetic variants. Bioinformatics. 2015;31(5):761-3.

5. MacArthur DG, Tyler-Smith C. Loss-of-function variants in the genomes of healthy humans. Hum Mol Genet. 2010;19(R2):125-30.

6. Robinson PN, Köhler S, Oellrich A, Project SMG, Wang K, Mungall CJ, Lewis SE, Washington N, Bauer S, Seelow D, Krawitz P, Gilissen C, Haendel M, Smedley D. Improved exome prioritization of disease genes through cross-species phenotype comparison. Genome Res. 2014;24(2): 340-8. https://doi.org/10.1101/gr.160325.113.

7. Boudellioua I, Mahamad Razali RB, Kulmanov M, Hashish Y, Bajic VB, Goncalves-Serra E, Schoenmakers N, Gkoutos GV, Schofield PN, Hoehndorf R. Semantic prioritization of novel causative genomic variants. PLOS Comput Biol. 2017;13(4):1-21. https://doi.org/10.1371/journal.pcbi. 1005500

8. Sifrim A, Popovic D, Tranchevent L-C, Ardeshirdavani A, Sakai R, Konings P, Vermeesch aR, Aerts J, De Moor B, Moreau Y. eXtasy: variant prioritization by genomic data fusion. Nat Methods. 2013;10:1083-4.

9. Singleton MV, Guthery SL, Voelkerding KV, Chen K, Kennedy B, Margraf RL, Durtschi J, Eilbeck K, Reese MG, Jorde LB, Huff CD, Yandell M. Phevor combines multiple biomedical ontologies for accurate identification of diseasecausing alleles in single individuals and small nuclear families. Am J Hum Genet. 2014;94(4):599-610. https://doi.org/10.1016/j.ajhg.2014.03.010.

10. de Bono B, Hoehndorf R, Wimalaratne S, Gkoutos GV, Grenon P. The ricordo approach to semantic interoperability for biomedical data and models: strategy, standards and solutions. BMC Res Notes. 2011;4(1):313.

11. Gkoutos GV, Green EC, Mallon A-MM, Hancock JM, Davidson D. Using ontologies to describe mouse phenotypes. Genome Biol. 2005;6(1):5. https://doi.org/10.1186/gb-2004-6-1-r8

12. de Angelis MH, Nicholson G, Selloum M, White JK, Morgan H, RamirezSolis R, Sorg T, Wells S, Fuchs H, Fray M, Adams DJ, Adams NC, Adler T, Aguilar-Pimentel A, Ali-Hadji D, Amann G, André P, Atkins S, Auburtin A, Ayadi A, Becker J, Becker L, Bedu E, Bekeredjian R, Birling M-C, Blake A, Bottomley J, Bowl MR, Brault V, Busch DH, Bussell JN, Calzada-Wack J, Cater H, Champy M-F, Charles P, Chevalier C, Chiani F, Codner GF, Combe R, Cox R, Dalloneau E, Dierich A, Fenza AD, Doe B, Duchon A, Eickelberg O, Esapa CT, Fertak LE, Feigel T, Emelyanova I, Estabel J, Favor J, Flenniken A, Gambadoro A, Garrett L, Gates H, Gerdin A-K, Gkoutos G, Greenaway S, Glasl L, GoetzP, Cruz IGD, Götz A, Graw J, Guimond A, Hans W, Hicks G, Hölter SM, Höfler H, Hancock JM, Hoehndorf R, Hough T, Houghton R, Hurt A, Ivandic B, Jacobs H, Jacquot S, Jones N, Karp NA, Katus HA, Kitchen S, Klein-Rodewald T, Klingenspor M, Klopstock T, Lalanne V, Leblanc S, Lengger C, le Marchand E, Ludwig T, Lux A, McKerlie C, Maier H, Mandel J-L, Marschall S, Mark M, Melvin DG, Meziane H, Micklich K, Mittelhauser C, Monassier L, Moulaert D, Muller S, Naton B, Neff F, Nolan PM, Nutter LMJ, Ollert M, Pavlovic G, Pellegata NS, Peter E, Petit-Demoulière B, Pickard A, Podrini C, Potter P, Pouilly L, Puk O, Richardson D, Rousseau S, Quintanilla-Fend L, Quwailid MM, Racz I, Rathkolb B, Riet F, Rossant J, Roux M, Rozman J, Ryder E, Salisbury J, Santos L, Schäble K-H, Schiller E, Schrewe A, Schulz H, Steinkamp R, Simon M, Stewart M, Stöger C, Stöger T, Sun M, Sunter D, Teboul L, Tilly I, Tocchini-Valentini GP, Tost M, Treise I, Vasseur L, Velot E, VogtWeisenhorn D, Wagner C, Walling A, Wattenhofer-Donze M, Weber B, Wendling $\mathrm{O}$, Westerberg $\mathrm{H}$, Willershäuser $\mathrm{M}$, Wolf $\mathrm{E}$, Wolter $\mathrm{A}$, Wood J, Wurst W, Önder Yildirim A, Zeh R, Zimmer A, Zimprich A, Holmes C, Steel KP, Herault Y, Gailus-Durner V, Mallon A-M, Brown SDM. Analysis of mammalian gene function through broad-based phenotypic screens across a consortium of mouse clinics. Nat Genet. 2015:47:969-978.

13. Amberger J, Bocchini C, Hamosh A. A new face and new challenges for Online Mendelian Inheritance in Man (OMIM). Hum Mutat. 2011;32:564-7.

14. Landrum MJ, Lee JM, Riley GR, Jang W, Rubinstein WS, Church DM, Maglott DR. Clinvar: public archive of relationships among sequence variation and human phenotype. Nucleic Acids Res. 2013. https://doi.org/ 10.1093/nar/gkt1113 http://nar.oxfordjournals.org/content/early/2013/ 11/14/nar.gkt1113.full.pdf+html.

15. Köhler S, Doelken SC, Ruef BJ, Bauer S, Washington N, Westerfield M, Gkoutos G, Schofield P, Smedley D, Lewis SE, Robinson PN, Mungall CJ. Construction and accessibility of a cross-species phenotype ontology along with gene annotations for biomedical research. F1000Research. 2013;2:. https://doi.org/10.12688/f1000research.2-30.v1.

16. Rodríguez-García MÁ, Gkoutos GV, Schofield PN, Hoehndorf R. Integrating phenotype ontologies with phenomenet. J Biomed Semant. 2017;8(1):58. https://doi.org/10.1186/s13326-017-0167-4

17. Smedley D, Robinson PN. Phenotype-driven strategies for exome prioritization of human mendelian disease genes. Genome Med. 2015;7(1):1-11. https://doi.org/10.1186/s13073-015-0199-2.

18. Smedley D, Schubach $M$, Jacobsen JOB, Köhler S, Zemojtel T, Spielmann M, Jäger M, Hochheiser $H$, Washington NL, McMurry JA, Haendel MA, Mungall CJ, Lewis SE, Groza T, Valentini G, Robinson PN. A Whole-Genome analysis framework for effective identification of pathogenic regulatory variants in mendelian disease. Am J Hum Genet. 2016;99(3):595-606. https://doi.org/10.1016/j.ajhg.2016.07.005.

19. Lecun $Y$, Bengio $Y$, Hinton G. Deep Learn. Nature. 2015;521(7553): 436-44. https://doi.org/10.1038/nature14539.

20. Grimm DG, Azencott C, Aicheler F, Gieraths U, MacArthur DG, Samocha KE, Cooper DN, Stenson PD, Daly MJ, Smoller JW, Duncan LE, Borgwardt KM The evaluation of tools used to predict the impact of missense variants is hindered by two types of circularity. Hum Mutat. 2015;36(5):513-23. https://doi.org/10.1002/humu.22768. https://onlinelibrary.wiley.com/doi/ pdf/10.1002/humu.22768

21. Cornish AJ, David A, Sternberg MJE. Phenorank: reducing study bias in gene prioritization through simulation. Bioinformatics. 2018:34:2087-2095.

22. Danecek P, Auton A, Abecasis G, Albers CA, Banks E, DePristo MA, Handsaker RE, Lunter G, Marth GT, Sherry ST, McVean G, Durbin R. The variant call format and vcftools. Bioinformatics. 2011;27(15):2156-8. https://doi.org/10.1093/bioinformatics/btr330.

23. Köhler S, Doelken SC, Mungall CJ, Bauer S, Firth HV, Bailleul-Forestier I, Black GCM, Brown DL, Brudno M, Campbell J, FitzPatrick DR, Eppig JT, Jackson AP, Freson K, Girdea M, Helbig I, Hurst JA, Jähn J, Jackson LG, Kelly AM, Ledbetter DH, Mansour S, Martin CL, Moss C, Mumford A, Ouwehand WH, Park S-M, Riggs ER, Scott RH, Sisodiya S, Vooren SV, Wapner RJ, Wilkie AOM, Wright CF, Vulto-van Silfhout AT, Leeuw Nd, de Vries BBA, Washingthon $\mathrm{NL}$, Smith $\mathrm{CL}$, Westerfield M, Schofield $\mathrm{P}$, Ruef BJ, Gkoutos GV, Haendel M, Smedley D, Lewis SE, Robinson PN. The human phenotype ontology project: linking molecular biology and disease through phenotype data. Nucleic Acids Res. 2014;42(D1):966-74.

24. The 1000 Genomes Project Consortium. A global reference for human genetic variation. Nature. 2015;526:68-74.

25. Quang D, Chen Y, Xie X. Dann: a deep learning approach for annotating the pathogenicity of genetic variants. Bioinformatics. 2015;31(5):761-3. https://doi.org/10.1093/bioinformatics/btu703. http://bioinformatics. oxfordjournals.org/content/31/5/761.full.pdf+html.

26. Ritchie GRS, Dunham I, Zeggini E, Flicek P. Functional annotation of noncoding sequence variants. Nat Methods. 2014;11:294-6.

27. Li H. Tabix: fast retrieval of sequence features from generic tab-delimited files. Bioinformatics. 2011;27 5:718-9.

28. Chollet F, et al. Keras. GitHub. 2015. https://github.com/keras-team/keras. Accessed 29 Jan 2019.

29. Abadi M, Agarwal A, Barham P, Brevdo E, Chen Z, Citro C, Corrado GS, Davis A, Dean J, Devin M, Ghemawat S, Goodfellow I, Harp A, Irving G, Isard M, Jia Y, Jozefowicz R, Kaiser L, Kudlur M, Levenberg J, Mané D, Monga R, Moore S, Murray D, Olah C, Schuster M, Shlens J, Steiner B, Sutskever I, Talwar K, Tucker P, Vanhoucke V, Vasudevan V, Viégas F, Vinyals $O$, Warden $P$, Wattenberg $M$, Wicke M, Yu Y, Zheng X TensorFlow: Large-Scale Machine Learning on Heterogeneous Systems. 2015. Software available from tensorflow.org. https://www.tensorflow. org/. Accessed 29 Jan 2019.

30. Pumperla M. Hyperas. GitHub. https://github.com/maxpumperla/ hyperas. Accessed 29 Jan 2019.

31. Bergstra J, Bardenet R, Bengio Y, Kégl B. Algorithms for hyper-paramete optimization. In: Proceedings of the 24th International Conference on Neural Information Processing Systems. NIPS'11. USA: Curran Associates Inc.; 2011. p. 2546-54. http://dl.acm.org/citation.cfm?id=2986459. 2986743 
32. Nair $\mathrm{V}$, Hinton GE. Rectified linear units improve restricted boltzmann machines. In: Proceedings of the 27th International Conference on International Conference on Machine Learning. ICML'10. USA: Omnipress; 2010. p. 807-14. http://dl.acm.org/citation.cfm?id=3104322.3104425.

33. Kingma DP, Ba J. Adam: A method for stochastic optimization. CoRR. 2014;abs/1412.6980:1412.6980. https://arxiv.org/abs/1412.6980.

34. Srivastava N, Hinton G, Krizhevsky A, Sutskever I, Salakhutdinov R. Dropout: A simple way to prevent neural networks from overfitting. J Mach Learn Res. 2014;15(1):1929-58.

35. Robinson PN, Köhler S, Bauer S, Seelow D, Horn D, Mundlos S. The human phenotype ontology: a tool for annotating and analyzing human hereditary disease. Am J Hum Genet. 2008;83(5):610-5. https://doi.org/10. 1016/j.j.jhg.2008.09.017.

36. Smith CL, Goldsmith C-AW, Eppig JT. The mammalian phenotype ontology as a tool for annotating, analyzing and comparing phenotypic information. Genome Biol. 2004;6(1):7. https://doi.org/10.1186/gb-20046-1-r7.

37. Fawcett T. An introduction to ROC analysis. Pattern Recogn Lett. 2006;27(8):861-74. https://doi.org/10.1016/j.patrec.2005.10.010.

38. Ball MP, Bobe JR, Chou MF, Clegg T, Estep PW, Lunshof JE, Vandewege W, Zaranek A, Church GM. Harvard personal genome project: lessons from participatory public research. Genome Med. 2014;6(2):10.

Ready to submit your research? Choose BMC and benefit from:

- fast, convenient online submission

- thorough peer review by experienced researchers in your field

- rapid publication on acceptance

- support for research data, including large and complex data types

- gold Open Access which fosters wider collaboration and increased citations

- maximum visibility for your research: over 100M website views per year

At $B M C$, research is always in progress.

Learn more biomedcentral.com/submission 